\title{
Super Characteristic Classes and Riemann-Roch Type Formula
}

\author{
Tadashi Taniguchi \\ Gunma National College of Technology, Maebashi-Shi, Japan \\ Email: tani@nat.gunma-ct.ac.jp
}

Received 26 March 2015; accepted 22 May 2015; published 25 May 2015

Copyright (C) 2015 by author and Scientific Research Publishing Inc.

This work is licensed under the Creative Commons Attribution International License (CC BY). http://creativecommons.org/licenses/by/4.0/

c) (i) Open Access

\begin{abstract}
The main purpose of this article is to define the super characteristic classes on a super vector bundle over a superspace. As an application, we propose the examples of Riemann-Roch type formula. We also introduce the helicity group and cohomology with respect to coefficient of the helicity group. As an application, we propose the examples of Gauss-Bonnet type formula.
\end{abstract}

\section{Keywords}

Superspace, Super Characteristic Class, Complex Supercurve with Genus g, SUSY Structure, Cohomology of Helicity Group

\section{Introduction}

In this paper, we define various characteristic classes on a super vector bundle over a superspace, so called super characteristic classes. We also propose the super Riemann-Roch formulas and the super Gauss-Bonnet formulas as its application. In contrast, it is justified the definition of the super characteristic classes by establishing those formulas. In [1], we defined the super Chern classes with values in the super number $(a \mid b), a, b \in \mathbb{Z}$ and we succeeded in applying the super ADHM construction of the super Yang-Mills instantons. But essentially the super Chern classes ought to take with values in an integer $a \in \mathbb{Z}$. Meaning like it, we introduce the new definition of the super Chern classes with values in integer. In general, the characteristic classes consider that given the vector bundles it corresponds to some cohomology class of the base manifolds. Hence, we need the cohomology reflecting the properties of superspaces. Therefore, we will define the cohomology with respect to coefficient of the some finitely generated group, which is called the helicity group.

This article is organized as follows. After a brief sketch on the definition and examples of superspaces and its cohomology in Section 2 ([1]-[6]), main result in this paper is that we define the Chern class, Chern character, Todd class, Pontrjagin class, Eular class, $\hat{A}$-genus and $L$-genus as in the case of super category in Section 3 . In 
Section 4, as an application, we have the Riemann-Roch type formula of super structure sheaf on the complex supercurves of dimension $(1 \mid N)$ with genus $g$. Moreover, it generalizes the structure sheaf to any super line sheaves. In particular, in the case of dimension (11), with $N=1$ supersymmetric structure, we obtain the Atiyah-Singer index type formula for any super line bundles. In Section 5, we attempt to define the helicity group and cohomology with respect to coefficient of the helicity group. In Section 6, we give the Gauss-Bonnet type formula on the complex supercurves of dimension $(1 \mid N)$ with genus $g$ and the complex super projectve space of dimension $(n \mid N)$.

\section{Supermanifolds}

We will summarize the definitions here in order to establish terminology and notation ([1]-[6]).

Definition 2.1 A superspace is defined to be a local ringed space $\hat{M}=\left(M, \hat{\mathcal{O}}_{M}\right)$ consisting a topological space $M$ and a sheaf of $\mathbb{Z}_{2}$-graded supercommutative rings $\hat{\mathcal{O}}_{M}=\hat{\mathcal{O}}_{M, \overline{0}} \oplus \hat{\mathcal{O}}_{M, \overline{1}}$ on it such that the stalk $\hat{\mathcal{O}}_{M, x}$ at any point $x \in M$ is a local ring.

In particular case of a superspace, a supermanifold is defined by the following.

Definition 2.2 A supermanifold of dimension $(n \mid N)$ is a ringed space $M=\left(M, \hat{\mathcal{O}}_{M}\right)$ with the following properties:

1) the structure sheaf $\hat{\mathcal{O}}_{M}=\hat{\mathcal{O}}_{M, \overline{0}} \oplus \hat{\mathcal{O}}_{M, \overline{1}}$ is a sheaf of $\mathbb{Z}_{2}$-graded supercommutative rings,

2) Let $\mathcal{N}_{\hat{M}}=\hat{\mathcal{O}}_{M, \overline{1}}+\hat{\mathcal{O}}_{M, \overline{1}}^{2}$ be the ideal sheaf of nilpotents in $\hat{\mathcal{O}}_{M}$. Then $M_{\text {red }}=\left(M, \mathcal{O}_{\text {red }}:=\hat{\mathcal{O}}_{M} / \mathcal{N}_{\hat{M}}\right)$ is a classical manifold $M$ of dimension $n$, so also called body.

3) Let $\mathcal{E}=\mathcal{N}_{\hat{M}} / \mathcal{N}_{\hat{M}}^{2}$ be the locally free $\mathcal{O}_{\text {red }}$-module of rank $(0 \mid N)$. Then $\hat{\mathcal{O}}_{M}$ is locally isomorphic to the exterior algebra $\Lambda \mathcal{E}$.

A supermanifold is said to be split if the isomorphism 3) holds globally.

A local section $f \in \Gamma\left(O_{M}\right)$ can be expressed as follows:

$$
f(z \mid \theta)=\sum_{k=01 \leq i_{1}<\cdots<i_{k} \leq N}^{N} f_{i_{1} \cdots i_{k}}(z) \theta^{i_{1}} \cdots \theta^{i_{k}}
$$

where $z=\left(z^{1}, z^{2}, \cdots, z^{n}\right), \quad f_{i_{1} \cdots i_{k}}(z)$ is a local coordinate function on $M_{\text {red }}$ and $\theta=\left(\theta^{1}, \theta^{2}, \cdots, \theta^{N}\right)$ a local generator of $\Lambda \mathcal{E}$. We refer to $\left(z_{1}, z_{2}, \cdots, z_{n} \mid \theta^{1}, \theta^{2}, \cdots, \theta^{N}\right)$ as a local coordinate of a supermanifold $\hat{M}$.

Example 2.1 1) The typical example is the real (or complex) linear superspace $\mathbb{R}^{n \mid N}$ (or $\mathbb{C}^{n \mid N}$ ) which can be defined by

$$
\begin{aligned}
& \mathbb{R}^{n \mid N}=\left(\mathbb{R}^{n}, \Lambda\left(\mathbb{R}^{N} \otimes_{\mathbb{R}} \mathcal{A}_{\mathbb{R}^{n}}\right)\right), \\
& \mathbb{C}^{n \mid N}=\left(\mathbb{C}^{n}, \Lambda\left(\mathbb{C}^{N} \otimes_{\mathbb{C}} \mathcal{A}_{\mathbb{C}^{n}}\right)\right),
\end{aligned}
$$

where $\mathcal{A}_{\mathbb{R}^{n}}$ (or $\mathcal{A}_{\mathbb{C}^{n}}$ ) is the sheaf of the ring of differential functions on $\mathbb{R}^{n}$ (or $\mathbb{C}^{n}$ ). It is easy to see that the $\mathbb{R}^{n \mid N}$ is isomorphic to $\left(\mathbb{R}^{n}, \mathcal{A}\left(S\left(\mathbb{R}^{n}\right) \otimes \Lambda \mathbb{R}^{N}\right)\right)$.

2) A real super sphere of dimension $(n \mid 2 N)$ is defined by

$$
S^{n \mid 2 N}=\left(S^{n}, \Lambda\left(\mathbb{R}^{2 N} \otimes_{\mathbb{R}} \mathcal{A}_{S^{n}}\right)\right),
$$

where $\mathcal{A}_{S^{n}}$ is the sheaf of the ring of differential functions on $S^{n}$.

3) A complex super projective space of dimensin $(n \mid N)$ is defined by

$$
P^{n \mid N}=\left(P^{n}, \Lambda\left(\mathbb{C}^{N} \otimes_{\mathbb{C}} \mathcal{O}_{P^{n}}(-1)\right)\right) .
$$

We denote by $\mathcal{O}_{p^{n \mid N}}=\hat{\mathcal{O}}_{p^{n}}$ the structure sheaf $\Lambda\left(\mathbb{C}^{N} \otimes_{\mathbb{C}} \mathcal{O}_{p^{n}}(-1)\right)$ of $P^{n \mid N}$. A super holomorphic function 1) on $P^{n \mid N}$ should be a function of total homogeneity 0 in $^{P^{n}} n+1$ even variables $\left(z^{1}, \cdots, z^{n+1}\right)$ and $N$ odd variables $\left(\theta^{1}, \cdots, \theta^{N}\right)$, that is $f_{i_{1} \cdots i_{k}}(z)$ has homogeneity $(-k)$. Let $\mathcal{O}_{p^{n \mid N}}(d)=\mathcal{O}_{p^{n}}(d) \otimes \mathcal{O}_{P^{n \mid N}}$ be the 
even line sheaf of degree $d$ on $P^{n \mid N}$ and $\Pi \mathcal{O}_{P^{n \mid N}}(d)=\Pi \mathcal{O}_{P^{n}}(d) \otimes \mathcal{O}_{P^{n \mid N}}$ be the odd line sheaf of degree $d$ on $P^{n \mid N}$.

4) A quaternionic super projective space of dimension $(n \mid N)$ is defined by

$$
\mathbb{H} P^{n \mid N}=\left(\mathbb{H} P^{n}, \Lambda\left(\mathbb{H}^{N} \otimes_{\mathbb{H}} \mathcal{A}_{\mathbb{H} P^{n}}(-1)\right)\right) .
$$

The above are examples of the supermanifolds in Definition 2.2.

5) We have a new example of superspace in Definition 2.1 as follows. The complex supercurves of dimension (1| $N)$ with genus $g$ is defined by

$$
\hat{\Sigma}=\left(\Sigma, \hat{\mathcal{O}}_{\Sigma}\right)=\left(\Sigma, \Lambda \cdot\left(\mathbb{C}^{N} \otimes K_{\Sigma}^{\frac{1}{2}}\right)\right),
$$

where $K_{\Sigma} \simeq T^{*} \Sigma$ is the canonical line bundle on the classical Riemann surfaces $\Sigma$ and $K_{\Sigma}^{-1} \simeq T \Sigma$. In the case of $N=1$, it becomes the super Riemann surfaces with $N=1$ SUSY structure (c.f. [7], p.162). In the case of $N \geq 2$, we do not kown whether or not there exists a SUSY structure.

We can construct the super Euler sequence as follows ([1]).

$$
0 \rightarrow \mathcal{O}_{P^{n \mid N}}(-1) \rightarrow P^{n \mid N} \times \mathbb{C}^{n+1 \mid N} \rightarrow T P^{n \mid N} \otimes \mathcal{O}_{P^{n \mid N}}(-1) \rightarrow 0 .
$$

Tensoring this with $\mathcal{O}_{p^{n \mid N}}(1)$, we have

$$
0 \rightarrow \mathcal{O}_{P^{n \mid N}} \rightarrow\left(P^{n \mid N} \times \mathbb{C}^{n+1 \mid N}\right) \otimes \mathcal{O}_{P^{n \mid N}}(1) \rightarrow T P^{n \mid N} \rightarrow 0 .
$$

Considering the super determinant ( so called Berezin bundle ) of the super Euler sequence, we obtain

$$
\operatorname{BerTP}^{n \mid N}=\Pi^{n+N+1} \mathcal{O}_{P^{n \mid N}}(n+1-N) .
$$

Dualizing this, we can write

$$
\operatorname{Ber}^{*} P^{n \mid N}=\Pi^{n+N+1} \mathcal{O}_{P^{n \mid N}}(-n-1+N),
$$

where $\operatorname{Ber} T^{*} P^{n \mid N}$ calls the canonical super line bundle of $P^{n \mid N}$ and $\Pi$ is the parity change functor. The following is given by Manin ([5]).

\section{Lemma 2.1}

$$
\begin{gathered}
H^{0}\left(P^{n \mid N}, \mathcal{O}_{P^{n \mid N}}(d)\right)= \begin{cases}S^{d}\left(\mathbb{C}^{n+1 \mid N}\right)^{*} & (d \geq 0) \\
0 & (d<0) .\end{cases} \\
H^{p}\left(P^{n \mid N}, \mathcal{O}_{P^{n \mid N}}(d)\right)=0, \quad p=1,2, \cdots, n-1 . \\
H^{n}\left(P^{n \mid N}, \mathcal{O}_{P^{n \mid N}}(d)\right)= \begin{cases}S^{-d+N-(n+1)}\left(\mathbb{C}^{n+1 \mid N}\right) \otimes \operatorname{Ber}\left(\mathbb{C}^{n+1 \mid N}\right)^{*} & (d \leq N-(n+1)) \\
0 & (d>N-(n+1)),\end{cases}
\end{gathered}
$$

where $\operatorname{Ber}\left(\mathbb{C}^{n \mid N}\right)^{*}=\Pi^{n+N} \mathbb{C}$ and $\mathcal{O}_{p^{n \mid N}}(d)=\mathcal{O}_{P^{n}}(d) \otimes \mathcal{O}_{p^{n \mid N}}$

The following is given by Penkov ([8]).

Theorem 2.1 (Super Serre Duality) Let $E$ be a complex super vector bundle over $\hat{M}$. Suppose that $\operatorname{Ber} T^{*} \hat{M}$ is the canonical super line bundle of $\hat{M}$. Then we have the following.

$$
H^{p}(\hat{M}, E)^{*} \simeq H^{n-p}\left(\hat{M}, E^{*} \otimes \operatorname{BerT}^{*} \hat{M}\right) .
$$

\section{Super Characteristic Class}

In this section, we will give a main result in this paper. Let $\hat{\mathcal{O}}_{M}=\hat{\mathcal{O}}_{M, \overline{0}} \oplus \hat{\mathcal{O}}_{M, \overline{1}}$ denote the structure sheaf on 
$\hat{M}$. Then we have an exact sequence (cf. [2], p.166 Lemma 2.1)

$$
0 \rightarrow Z \stackrel{\iota}{\longrightarrow} \hat{\mathcal{O}}_{M, \overline{0}} \stackrel{\exp }{\longrightarrow} \hat{\mathcal{O}}_{M, \overline{0}}^{*} \rightarrow 1,
$$

where $l$ is the natural injection and exp is defined by

$$
\exp (f)=\exp (2 \pi i f) \text { for } f \in \hat{\mathcal{O}}_{M, \overline{0}} .
$$

The $\exp (f)=1$ implies $f=l \in \mathbb{Z}, l \in \mathbb{Z}$. Hence $\operatorname{Ker}(\exp )=\mathbb{Z}$. This induces the exact sequence of cohomology groups:

$$
\cdots \rightarrow H^{1}\left(\hat{M}, \hat{\mathcal{O}}_{M, \overline{0}}\right) \stackrel{e}{\longrightarrow} H^{1}\left(\hat{M}, \hat{\mathcal{O}}_{M, \overline{0}}^{*}\right) \stackrel{\delta}{\longrightarrow} H^{2}(\hat{M}, \mathbb{Z}) \rightarrow \cdots
$$

We can identify $H^{1}\left(\hat{M}, \hat{\mathcal{O}}_{M, \overline{0}}^{*}\right)$ with the equivalence classes of $(1 \mid 0)$ or $(0 \mid 1)$-super line bundles over $\hat{M}$. Then we can define the super first Chern class of (1|0)-super line bundle $L$ and (0|1)-super line bundle $\Pi L$ by

$$
c_{1}(L)=\delta(L), \quad-c_{1}(L)=\delta(\Pi L), \quad L, \Pi L \in H^{1}\left(\hat{M}, \hat{\mathcal{O}}_{M, \overline{0}}^{*}\right) .
$$

Remark 3.1 Note that we can define $\mathbb{Z}_{N}=\Lambda \mathbb{Z}^{N}$. We consider the line sheaf $\mathcal{O}_{P^{n / 2}}(d)$ over the complex super projective space $P^{n \mid 2}$. This line sheaf is decomposed into

$$
\mathcal{O}_{p^{n / 2}}(d)=\mathcal{O}_{p^{n}}(d)+\mathcal{O}_{p^{n}}(d-1) \theta^{1}+\mathcal{O}_{p^{n}}(d-1) \theta^{2}+\mathcal{O}_{p^{n}}(d-2) \theta^{1} \theta^{2}
$$

The super first Chern calss and the classical first Chern class denote by $c_{1}$ and $\tilde{c}_{1}$, respectively. Then we have

$$
\begin{gathered}
c_{1}\left(\mathcal{O}_{p^{n \mid 2}}(d)\right)=d \in \mathbb{Z}, \\
\tilde{c}_{1}\left(\mathcal{O}_{P^{n / 2}}(d)\right)=d+(d-1) \theta^{1}+(d-1) \theta^{2}+(d-2) \theta^{1} \theta^{2} \in \mathbb{Z}_{N=2} .
\end{gathered}
$$

Hence, we see that for the superline bundle $L$

$$
c_{1}(L) \in H^{2}(\hat{M}, \mathbb{Z}), \quad \tilde{c}_{1}(L) \in H^{2}\left(\hat{M}, \mathbb{Z}_{N}\right) .
$$

We will propose the axiomatic definition of super Chern classes (cf. [1] [2] [9]-[15]). We consider the category of complex $(r \mid s)$-super vector bundles over an $(n \mid N)$-superspace $\hat{M}=\left(M, \hat{\mathcal{O}}_{M}\right)$.

Axiom 1 For each complex super vector bundle $E$ over $\hat{M}$ and for each positive integer $i$, the $i$-th super Chern class $c_{i}(E) \in H^{2 i}(\hat{M}, \mathbb{Z})$ is given, and $c_{0}(E)=1$.

We set $c(E)=\sum_{i=0}^{\infty} c_{i}(E)$ and call $c(E)$ the total super Chern class of $E$.

\section{Axiom 2 (Naturality)}

Let $E$ be a complex super vector bundle over a superspace $\hat{N}$ and $f:\left(M, \hat{\mathcal{O}}_{M}\right) \rightarrow\left(N, \hat{\mathcal{O}}_{N}\right)$ a morphism of superspaces. Then

$$
c\left(f^{*} E\right)=f^{*}(c(E)) \in H^{*}(\hat{M}, \mathbb{Z}),
$$

where $f^{*} E$ is the pull-back bundle over $\hat{M}$.

\section{Axiom 3 (Whitney sum formula)}

Let $L_{1}, L_{2}, \cdots, L_{q}$ be complex line bundles of rank $(1 \mid 0)$ or $(0 \mid 1)$ and $L_{1} \oplus L_{2} \oplus \cdots \oplus L_{q}$ be their Whitney sum. Then

$$
c\left(L_{1} \oplus L_{2} \oplus \cdots \oplus L_{q}\right)=c\left(L_{1}\right) \cdot c\left(L_{2}\right) \cdots c\left(L_{q}\right) .
$$

\section{Axiom 4 (Normalization)}

We put $\quad c\left(\mathcal{O}_{P^{n \mid N}}(1)\right)=1+c_{1}\left(\mathcal{O}_{P^{n \mid N}}(1)\right) \in H^{*}\left(P^{n}, \mathbb{Z}\right) \quad$ and $\quad c\left(\Pi \mathcal{O}_{P^{n \mid N}}(1)\right)=1+c_{1}\left(\Pi \mathcal{O}_{P^{n \mid N}}(1)\right) \in H^{*}\left(P^{n}, \mathbb{Z}\right)$. 
Then it can be axiomatically as follows:

$$
\begin{gathered}
c_{1}\left(\mathcal{O}_{P^{n \mid N}}(1)\right)=1 \in H^{2}\left(P^{n}, \mathbb{Z}\right) \simeq \mathbb{Z}, \\
c_{1}\left(\Pi \mathcal{O}_{P^{n \mid N}}(1)\right)=c_{1}\left(\mathcal{O}_{P^{n \mid N}}(-1)\right)-1 \in H^{2}\left(P^{n}, \mathbb{Z}\right) \simeq \mathbb{Z}, \\
c_{1}\left(\mathcal{O}_{P^{n \mid N}}\right)=c_{1}\left(\Pi \mathcal{O}_{P^{n \mid N}}\right)=0 .
\end{gathered}
$$

In order to explicitly define the super characteristic classes we need the splitting principle ([2] Proposition 3.7) as follows.

Proposition 3.1 (Bartocci, Bruzzo, Hernandez-Ruiperez) Let E be a complex $(r \mid s)$-super vector bundle over an $(n \mid N)$-supermanifold $\hat{M}$. Then there exists a supermanifold $F(\hat{M})$ and a proper fibration $\pi: F(\hat{M}) \rightarrow \hat{M}$ such that

1) The homomorphism $\pi^{*}: H^{*}(\hat{M}, \mathbb{Z}) \rightarrow H^{*}(F(\hat{M}), \mathbb{Z})$ is injective.

2) The pull-back bundle $\pi^{*} E$ splits into a direct sum of even complex line bundles $l_{i}$ of rank $(1 \mid 0)$ and odd complex line bundles $m_{j}$ of rank $(0 \mid 1)$ :

$$
\begin{aligned}
& \pi^{*} E \simeq l_{1} \oplus \cdots \oplus l_{r} \oplus m_{1} \oplus \cdots \oplus m_{s}, \\
& \operatorname{sdet}\left(\pi^{*} E\right) \simeq l_{1} \otimes \cdots \otimes l_{r} \otimes m_{1}^{-1} \otimes \cdots \otimes m_{s}^{-1} .
\end{aligned}
$$

We will explicitly give the super characteristic classes.

Definition 3.11 ) The total super Chern class $c(E) \in H^{*}(\hat{M}, \mathbb{Z})$ is defined by

$$
c(E)=\prod_{j=1}^{r}\left(1+\gamma_{j}\right) \prod_{k=1}^{s}\left(1-\delta_{k}\right), \quad \gamma_{j}, \delta_{k} \in H^{2}(\hat{M}, \mathbb{Z}) .
$$

2) The total super Chern character $\operatorname{ch}(E) \in H^{*}(\hat{M}, \mathbb{Q})$ is defined by

$$
\operatorname{ch}(E)=\sum_{j=1}^{r} \mathrm{e}^{\gamma_{j}}+\sum_{k=1}^{s} \mathrm{e}^{-\delta_{k}} .
$$

3) The super Todd class $t d(E) \in H^{*}(\hat{M}, \mathbb{Q})$ is defined by

$$
\operatorname{td}(E)=\prod_{i=1}^{r} \frac{\gamma_{i}}{1-\mathrm{e}^{-\gamma_{i}}} \prod_{j=1}^{s} \frac{-\delta_{j}}{1-\mathrm{e}^{\delta_{j}}} .
$$

4) The super Eular class $e(E)$ is defined by

$$
e(E)=c_{r+s}(E) \quad(r+s \leq n) \quad \text { or } \quad e(E)=c_{n}(E) \quad(r+s>n)
$$

5) Let $E_{\mathbb{R}}$ be a real vector bundle of rank $(2 r \mid 2 s)$. The $i$-th super Pontrjagin class $p_{i}\left(E_{\mathbb{R}}\right)$ and the total super Pontrjagin class are defined by

$$
\begin{aligned}
& p_{i}\left(E_{\mathbb{R}}\right)=(-1)^{i} c_{2 i}\left(E_{\mathbb{R}} \otimes \mathbb{C}\right), \\
& p\left(E_{\mathbb{R}}\right)=\prod_{j=1}^{r}\left(1+\gamma_{j}^{2}\right) \prod_{k=1}^{s}\left(1+\delta_{k}^{2}\right) .
\end{aligned}
$$

6) The super $\hat{A}$-genus $\hat{A}\left(E_{\mathbb{R}}\right) \in H^{*}(\hat{M}, \mathbb{Q})$ is defined by

$$
\hat{A}\left(E_{\mathbb{R}}\right)=\prod_{j=1}^{r} \frac{\gamma_{j} / 2}{\sinh \left(\gamma_{j} / 2\right)} \prod_{k=1}^{s} \frac{\delta_{k} / 2}{\sinh \left(\delta_{k} / 2\right)} .
$$

7) The super $L$-genus $L\left(E_{\mathbb{R}}\right) \in H^{*}(\hat{M}, \mathbb{Q})$ is defined by 


$$
L\left(E_{\mathbb{R}}\right)=\prod_{j=1}^{r} \frac{\gamma_{j}}{\tanh \gamma_{j}} \prod_{k=1}^{s} \frac{\delta_{k}}{\tanh \delta_{k}} .
$$

We can consider that it is justified these definitions by the following (cf. [13] [14]).

Lemma 3.1 The first few terms of $\operatorname{ch}(E)$ and $t d(E)$ are given by the following.

$$
\begin{aligned}
& c h_{0}(E)=r+s, \quad c h_{1}(E)=c_{1}(E), \quad c h_{2}(E)=\frac{1}{2}\left(c_{1}(E)^{2}-2 c_{2}(E)\right), \\
& c h_{3}(E)=\frac{1}{6}\left(c_{1}(E)^{3}-3 c_{1}(E) c_{2}(E)+3 c_{3}(E)\right), \quad t d_{0}(E)=1, \quad t d_{1}(E)=\frac{1}{2} c_{1}(E), \\
& t d_{2}(E)=\frac{1}{12}\left(c_{1}(E)^{2}+c_{2}(E)\right), \quad t d_{3}(E)=\frac{1}{24} c_{1}(E) c_{2}(E) .
\end{aligned}
$$

Proof. Let $E$ be a complex rank-(2|1) super vector bundle over a complex (4|4)-dimensional supermanifold $\hat{M}$. Then, total super Chern class is written by

$$
c(E)=\left(1+\gamma_{1}\right)\left(1+\gamma_{2}\right)\left(1-\delta_{1}\right) .
$$

Hence, we have

$$
\begin{aligned}
& c_{1}(E)=\gamma_{1}+\gamma_{2}-\delta_{1} \in H^{2}(\hat{M}, \mathbb{Z}), \\
& c_{2}(E)=\gamma_{1} \gamma_{2}-\gamma_{1} \delta_{1}-\gamma_{2} \delta_{1} \in H^{4}(\hat{M}, \mathbb{Z}), \\
& c_{3}(E)=-\gamma_{1} \gamma_{2} \delta_{1} \in H^{6}(\hat{M}, \mathbb{Z}) .
\end{aligned}
$$

The total super Chern character is written by

$$
\operatorname{ch}(E)=\mathrm{e}^{\gamma_{1}}+\mathrm{e}^{\gamma_{2}}+\mathrm{e}^{-\delta_{1}} .
$$

Hence we have

$$
\begin{aligned}
& c h_{0}(E)=3 \in H^{0}(\hat{M}, \mathbb{Q}), \\
& \operatorname{ch}_{1}(E)=\gamma_{1}+\gamma_{2}-\delta_{1} \in H^{2}(\hat{M}, \mathbb{Q}), \\
& \operatorname{ch}_{2}(E)=\frac{1}{2 !}\left(\gamma_{1}^{2}+\gamma_{2}^{2}+\delta_{1}^{2}\right) \in H^{4}(\hat{M}, \mathbb{Q}), \\
& \operatorname{ch}_{3}(E)=\frac{1}{3 !}\left(\gamma_{1}^{3}+\gamma_{2}^{3}-\delta_{1}^{3}\right) \in H^{6}(\hat{M}, \mathbb{Q}) .
\end{aligned}
$$

It is well-known thtat

$$
\begin{aligned}
& \frac{x}{1-\mathrm{e}^{-x}}=1+\frac{1}{2} x+\frac{1}{12} x^{2}+0 x^{3}-\frac{1}{720} x^{4}+\cdots, \\
& \frac{-x}{1-\mathrm{e}^{x}}=1-\frac{1}{2} x+\frac{1}{12} x^{2}+0 x^{3}-\frac{1}{720} x^{4}+\cdots
\end{aligned}
$$

Hence the total super Todd class is written by

$$
\operatorname{td}(E)=\left(1+\frac{1}{2} \gamma_{1}+\frac{1}{12} \gamma_{1}^{2}\right)\left(1+\frac{1}{2} \gamma_{2}+\frac{1}{12} \gamma_{2}^{2}\right)\left(1-\frac{1}{2} \delta_{1}+\frac{1}{12} \delta_{1}^{2}\right) .
$$


Therefore we have

$$
\begin{aligned}
& t d_{0}(E)=1 \in H^{0}(\hat{M}, \mathbb{Q}), \\
& t d_{1}(E)=\frac{1}{2}\left(\gamma_{1}+\gamma_{2}-\delta_{1}\right) \in H^{2}(\hat{M}, \mathbb{Q}), \\
& t d_{2}(E)=\frac{1}{12}\left(3 \gamma_{1} \gamma_{2}-3 \gamma_{1} \delta_{1}-3 \gamma_{2} \delta_{1}+\gamma_{1}^{2}+\gamma_{2}^{2}+\delta_{1}^{2}\right) \in H^{4}(\hat{M}, \mathbb{Q}), \\
& t_{3}(E)=\frac{1}{24}\left(\gamma_{1} \gamma_{2}^{2}+\gamma_{1}^{2} \gamma_{2}+\gamma_{1} \delta_{1}^{2}-\gamma_{1}^{2} \delta_{1}+\gamma_{2} \delta_{1}^{2}-\gamma_{2}^{2} \delta_{1}-3 \gamma_{1} \gamma_{2} \delta_{1}\right) \in H^{6}(\hat{M}, \mathbb{Q}) .
\end{aligned}
$$

Then, they satisfy that

$$
\begin{aligned}
& c h_{0}(E)=r+s, \quad c h_{1}(E)=c_{1}(E), \quad c h_{2}(E)=\frac{1}{2}\left(c_{1}(E)^{2}-2 c_{2}(E)\right), \\
& c h_{3}(E)=\frac{1}{6}\left(c_{1}(E)^{3}-3 c_{1}(E) c_{2}(E)+3 c_{3}(E)\right), \quad t d_{0}(E)=1, \quad t d_{1}(E)=\frac{1}{2} c_{1}(E), \\
& t d_{2}(E)=\frac{1}{12}\left(c_{1}(E)^{2}+c_{2}(E)\right), \quad t d_{3}(E)=\frac{1}{24} c_{1}(E) c_{2}(E) .
\end{aligned}
$$

Lemma 3.2 The first few terms of $\hat{A}\left(E_{\mathbb{R}}\right), L\left(E_{\mathbb{R}}\right)$ and $p\left(E_{\mathbb{R}}\right)$ are given by the following.

$$
\begin{aligned}
& \hat{A}_{0}\left(E_{\mathbb{R}}\right)=1, \quad \hat{A}_{1}\left(E_{\mathbb{R}}\right)=-\frac{1}{24} p_{1}\left(E_{\mathbb{R}}\right), \quad \hat{A}_{2}\left(E_{\mathbb{R}}\right)=\frac{1}{2^{7} \times 3^{2} \times 5}\left(7 p_{1}\left(E_{\mathbb{R}}\right)^{2}-4 p_{2}\left(E_{\mathbb{R}}\right)\right), \cdots, \\
& L_{0}\left(E_{\mathbb{R}}\right)=1, \quad L_{1}\left(E_{\mathbb{R}}\right)=\frac{1}{3} p_{1}\left(E_{\mathbb{R}}\right), \quad L_{2}\left(E_{\mathbb{R}}\right)=\frac{1}{45}\left(-p_{1}\left(E_{\mathbb{R}}\right)^{2}+7 p_{2}\left(E_{\mathbb{R}}\right)\right), \cdots, \\
& p_{1}\left(E_{\mathbb{R}}\right)=c_{1}(E)^{2}-2 c_{2}(E), \quad p_{2}\left(E_{\mathbb{R}}\right)=c_{2}(E)^{2}-2 c_{1}(E) c_{3}(E)+2 c_{4}(E) .
\end{aligned}
$$

Proof. $\hat{A}\left(E_{\mathbb{R}}\right), L\left(E_{\mathbb{R}}\right)$ and $p\left(E_{\mathbb{R}}\right)$ similarly form in the classical case. Therefore $\hat{A}\left(E_{\mathbb{R}}\right)$ and $L\left(E_{\mathbb{R}}\right)$ are of same argument (cf. [13]).

Let $E$ be a complex rank-(2|2) super vector bundle over a complex (4|6)-dimensional supermanifold $\hat{M}$. The total super Chern class is written by

$$
c(E)=\left(1+\gamma_{1}\right)\left(1+\gamma_{2}\right)\left(1-\delta_{1}\right)\left(1-\delta_{2}\right) .
$$

Hence, we have

$$
\begin{aligned}
& c_{1}(E)=\gamma_{1}+\gamma_{2}-\delta_{1}-\delta_{2} \in H^{2}(\hat{M}, \mathbb{Z}), \\
& c_{2}(E)=\gamma_{1} \gamma_{2}-\gamma_{1} \delta_{1}-\gamma_{1} \delta_{2}-\gamma_{2} \delta_{1}-\gamma_{2} \delta_{2}+\delta_{1} \delta_{2} \in H^{4}(\hat{M}, \mathbb{Z}), \\
& c_{3}(E)=-\gamma_{1} \gamma_{2} \delta_{1}-\gamma_{1} \gamma_{2} \delta_{2}+\gamma_{1} \delta_{1} \delta_{2}+\gamma_{2} \delta_{1} \delta_{2} \in H^{6}(\hat{M}, \mathbb{Z}), \\
& c_{4}(E)=\gamma_{1} \gamma_{2} \delta_{1} \delta_{2} \in H^{8}(\hat{M}, \mathbb{Z}) .
\end{aligned}
$$

The total super Pontrjagin class is written by

$$
p\left(E_{\mathbb{R}}\right)=\left(1+\gamma_{1}^{2}\right)\left(1+\gamma_{2}^{2}\right)\left(1+\delta_{1}^{2}\right)\left(1+\delta_{2}^{2}\right) .
$$

Hence, we have 


$$
\begin{aligned}
& p_{1}\left(E_{\mathbb{R}}\right)=\gamma_{1}^{2}+\gamma_{2}^{2}+\delta_{1}^{2}+\delta_{2}^{2} \in H^{4}(\hat{M}, \mathbb{Z}), \\
& p_{2}\left(E_{\mathbb{R}}\right)=\gamma_{1}^{2} \gamma_{2}^{2}+\gamma_{1}^{2} \delta_{1}^{2}+\gamma_{1}^{2} \delta_{2}^{2}+\gamma_{2}^{2} \delta_{1}^{2}+\gamma_{2}^{2} \delta_{2}^{2}+\delta_{1}^{2} \delta_{2}^{2} \in H^{8}(\hat{M}, \mathbb{Z}) .
\end{aligned}
$$

Then, they satisfy that

$$
p_{1}\left(E_{\mathbb{R}}\right)=c_{1}(E)^{2}-2 c_{2}(E), \quad p_{2}\left(E_{\mathbb{R}}\right)=c_{2}(E)^{2}-2 c_{1}(E) c_{3}(E)+2 c_{4}(E) .
$$

\section{Riemann-Roch Type Formula}

Let $\hat{\Sigma}=\left(\Sigma, \hat{\mathcal{O}}_{\Sigma}\right)=\left(\Sigma, \Lambda \cdot\left(\mathbb{C}^{N} \otimes K_{\Sigma}^{\frac{1}{2}}\right)\right)$ be the complex supercurves with genus $g$, where $K_{\Sigma} \simeq T^{*} \Sigma, K_{\Sigma}^{-1} \simeq T \Sigma$, in Example 2.1 (5). Then the canonical super line bundle on $\hat{\Sigma}$ is explicitly written by

$$
K_{\hat{\Sigma}}=\operatorname{Ber}^{*} \hat{\Sigma}=\hat{\mathcal{O}}_{\Sigma} \otimes\left(T^{*} \Sigma \otimes K_{\Sigma}^{-\frac{1}{2}} \otimes \cdots \otimes K_{\Sigma}^{-\frac{1}{2}}\right)=\hat{\mathcal{O}}_{\Sigma} \otimes\left(K_{\Sigma}^{1-\frac{N}{2}}\right) .
$$

Hence we have $c_{1}\left(K_{\hat{\Sigma}}\right)=\left(1-\frac{N}{2}\right)(2 g-2)=(2-N)(g-1)$.

Note that for any object $E$ and $F$ the parity change functor $\Pi$ satisfies

$$
\Pi E \oplus \Pi F=\Pi(E \oplus F), \quad \Pi E \otimes \Pi F=\Pi^{2}(E \otimes F)=E \otimes F .
$$

In general, if $\hat{M}=(M, \Lambda \mathcal{E})$ is a supermanifold, then its tangent bundle can be written by $T \hat{M}=\hat{\mathcal{O}}_{M} \otimes\left(T M \oplus \mathcal{E}^{*}\right)$ (cf. [16]). Hence we have

$$
T \hat{\Sigma} \simeq \hat{\mathcal{O}}_{\Sigma} \otimes\left(T \Sigma \oplus\left[K_{\Sigma}^{-\frac{1}{2}}\right]^{\oplus N}\right) .
$$

Using this decomposition, Euler number of $T \hat{\Sigma}$ get

$$
e(T \hat{\Sigma})=c_{1}(T \hat{\Sigma})=c_{1}(T \Sigma)-c_{1}\left(\left[K_{\Sigma}^{-\frac{1}{2}}\right]^{\oplus N}\right)=(2-2 g)-N(1-g)=(2-N)(1-g)
$$

Note that $c_{1}\left(K_{\hat{\Sigma}}\right)=-c_{1}(T \hat{\Sigma})$.

Theorem 4.1 Let $\hat{\Sigma}$ be the complex $(1 \mid N)$-dimensional supercurves with genus $g$. Then, we have a Noether type formula as follows.

$$
\operatorname{dim} H^{0}\left(\hat{\Sigma}, O_{\Sigma}\right)-\operatorname{dim} H^{1}\left(\hat{\Sigma}, O_{\Sigma}\right)=2^{N-1} e(T \hat{\Sigma})=2^{N} t d_{1}(T \hat{\Sigma}) .
$$

Proof. Let $g=\operatorname{dim} H^{0}\left(\Sigma, K_{\Sigma}\right)$ be the genus on the classical Riemann surfaces and $q=\operatorname{dim} H^{0}\left(\Sigma, K_{\Sigma}^{\frac{1}{2}}\right)$ be the number of linear independent Dirac zero modes or harmonic spinors which is not topologically invariant.

The structure sheaf of the complex supercurves have decomposition

$$
\hat{\mathcal{O}}_{\Sigma}=\Lambda\left(K_{\Sigma}^{\frac{1}{2}}\right)^{\oplus N}=\mathcal{O}_{\Sigma}+N \Pi K_{\Sigma}^{\frac{1}{2}}+\left(\begin{array}{c}
N \\
2
\end{array}\right) K_{\Sigma}+\left(\begin{array}{c}
N \\
3
\end{array}\right) \Pi K_{\Sigma}^{\frac{3}{2}}+\cdots+\Pi^{N} K_{\Sigma}^{\frac{N}{2}} .
$$

In the case of genus $g \geq 2$, we have (cf. ([17])) 


$$
\operatorname{dim} H^{0}\left(\Sigma, K_{\Sigma}^{\frac{p}{2}}\right)= \begin{cases}(p-1)(g-1) & (p \geq 3) \\ 1 & (p=0) \\ 0 & (p<0)\end{cases}
$$

In the case of genus $g=1$, it always satisfies $\operatorname{dim} H^{0}\left(T^{11 N}, K_{\Sigma}^{\frac{p}{2}}\right)=1$ for any $p$. In the case of genus $g=0$, it satisfies

$$
\operatorname{dim} H^{0}\left(P^{1 \mid N}, K_{\Sigma}^{\frac{p}{2}}\right)= \begin{cases}(1-p) & (p \leq 0) \\ 0 & (p>0)\end{cases}
$$

In the case of genus $g \geq 2$, we have the following.

$$
\begin{aligned}
\operatorname{dim} H^{0}\left(\hat{\Sigma}, \Lambda \cdot\left(K_{\Sigma}^{\frac{1}{2}}\right)^{\oplus N}\right)= & \operatorname{dim} H^{0}\left(\Sigma, \mathcal{O}_{\Sigma}\right)+\operatorname{dim} H^{0}\left(\Sigma, N K_{\Sigma}^{\frac{1}{2}}\right)+\operatorname{dim} H^{0}\left(\Sigma,\left(\begin{array}{c}
N \\
2
\end{array}\right) K_{\Sigma}\right) \\
& +\operatorname{dim} H^{0}\left(\Sigma,\left(\begin{array}{c}
N \\
3
\end{array}\right) K_{\Sigma}^{\frac{3}{2}}\right)+\cdots+\operatorname{dim} H^{0}\left(\Sigma, K_{\Sigma}^{\frac{N}{2}}\right) \\
= & 1+N q+\left(\begin{array}{c}
N \\
2
\end{array}\right) g+\sum_{m=3}^{N}\left(\begin{array}{l}
N \\
m
\end{array}\right)(m-1)(g-1) \\
\operatorname{dim} H^{1}\left(\hat{\Sigma}, \Lambda \cdot\left(K_{\Sigma}^{\frac{1}{2}}\right)^{\oplus N}\right)= & \operatorname{dim} H^{1}\left(\Sigma, \mathcal{O}_{\Sigma}\right)+\operatorname{dim} H^{1}\left(\Sigma, N K_{\Sigma}^{\frac{1}{2}}\right)+\operatorname{dim} H^{1}\left(\Sigma,\left(\begin{array}{c}
N \\
2
\end{array}\right) K_{\Sigma}\right) \\
& +\operatorname{dim} H^{1}\left(\Sigma,\left(\begin{array}{c}
N \\
3
\end{array}\right) K_{\Sigma}^{\frac{3}{2}}\right)+\cdots+\operatorname{dim} H^{1}\left(\Sigma, K_{\Sigma}^{\frac{N}{2}}\right) \\
\simeq & \operatorname{dim} H^{0}\left(\Sigma, K_{\Sigma}\right)+\operatorname{dim} H^{0}\left(\Sigma, N K_{\Sigma}^{\frac{1}{2}}\right)+\operatorname{dim} H^{0}\left(\Sigma,\left(\begin{array}{c}
N \\
2
\end{array}\right) O_{\Sigma}\right) \\
& +\operatorname{dim} H^{0}\left(\Sigma,\left(\begin{array}{l}
N \\
3
\end{array}\right) K_{\Sigma}^{\frac{-1}{2}}\right)+\cdots+\operatorname{dim} H^{0}\left(\Sigma, K_{\Sigma}^{\frac{2-N}{2}}\right) \\
= & g+N q+\left(\begin{array}{c}
N \\
2
\end{array}\right)
\end{aligned}
$$

Note that equal of second make use of the classical Serre duality. Hence we obtain

$$
\begin{aligned}
\operatorname{dim} H^{0}\left(\hat{\Sigma}, \Lambda\left(K_{\Sigma}^{\frac{1}{2}}\right)^{\oplus N}\right)-\operatorname{dim} H^{1}\left(\hat{\Sigma}, \Lambda \cdot\left(K_{\Sigma}^{\frac{1}{2}}\right)^{\oplus N}\right) & =1-g+\left(\begin{array}{c}
N \\
2
\end{array}\right)(g-1)+\sum_{m=3}^{N}\left(\begin{array}{c}
N \\
m
\end{array}\right)(m-1)(g-1) \\
& =\left(\left(\begin{array}{c}
N \\
2
\end{array}\right)-1+\sum_{m=3}^{N}\left(\begin{array}{c}
N \\
m
\end{array}\right)(m-1)\right)(g-1) \\
& =2^{N-1}(2-N)(1-g)=2^{N-1} e(T \hat{\Sigma})=2^{N} t d_{1}(T \hat{\Sigma}) .
\end{aligned}
$$

In the case of genus $g=1$ and $g=0$, we can prove similarly.

Corollary 4.1 Let $\hat{\Sigma}$ be the complex $(1 \mid N)$-dimensional supercurves with genus g. Then we have a RiemannRoch type formula as follows.

$$
\operatorname{dim} H^{0}\left(\hat{\Sigma}, \hat{\mathcal{O}}_{\Sigma}\right)-\operatorname{dim} H^{1}\left(\hat{\Sigma}, \hat{\mathcal{O}}_{\Sigma}\right)=2^{N}\left(\operatorname{ch}\left(\hat{\mathcal{O}}_{\Sigma}\right) \cdot t d(T \hat{\Sigma})\right)[\Sigma]
$$


where $[\Sigma] \in H_{2}(\Sigma, \mathbb{Z})$ is the fundamental homology class.

Proof.

$$
\begin{aligned}
\text { right hand side } & =2^{N}\left(\operatorname{ch}_{0}\left(\hat{\mathcal{O}}_{\Sigma}\right) \cdot t d_{1}(T \hat{\Sigma})+c h_{1}\left(\hat{\mathcal{O}}_{\Sigma}\right) \cdot t d_{0}(T \hat{\Sigma})\right) \\
& =2^{N}\left(1 \times \frac{1}{2} c_{1}(T \hat{\Sigma})+0 \times 1\right)=2^{N-1} c_{1}(T \hat{\Sigma})=2^{N-1} e(T \hat{\Sigma})=\text { left hand side }
\end{aligned}
$$

From Theorem 4.1, this completes the proof of Corollary 4.1.

The following Corollary essentially has been obtained by [18]. It needs the $N=1$ supersymmetric structure on the $N=1$ super Riemann surfaces (cf. [7] [19] [20]). The following rewrite the result of [21] to the super characteristic classes.

Corollary 4.2 Let $\hat{\Sigma}$ be the complex (1|1)-dimensional super Riemann surfaces and $L_{\hat{\Sigma}}=L_{\Sigma} \otimes \hat{\mathcal{O}}_{\Sigma}$ be any super line bundles of rank $(1 \mid 0)$ on $\hat{\Sigma}$. Then we have a Atiyah-Singer index type formula as follows.

$$
\operatorname{dimKer} \hat{\bar{\partial}}_{L_{\hat{\Sigma}}}-\operatorname{dimCoker} \hat{\bar{\partial}}_{L_{\hat{\Sigma}}}=2\left(\operatorname{ch}\left(L_{\hat{\Sigma}}\right) \cdot \operatorname{td}(T \hat{\Sigma})\right)[\Sigma],
$$

where $[\Sigma] \in H_{2}(\Sigma, \mathbb{Z})$ is the fundamental homology class.

Proof. The canonical super line bundle $K_{\hat{\Sigma}}$ of a super Riemann surface $\hat{\Sigma}$ can be defined by splitting the Berezin bundle $\operatorname{Ber} T^{*} \hat{\Sigma}$ using the super complex structure $\operatorname{Ber} T^{*} \hat{\Sigma} \simeq K_{\hat{\Sigma}} \otimes \bar{K}_{\hat{\Sigma}}$. We get an exact sequence ([21] [22])

$$
0 \rightarrow \mathbb{C} \hookrightarrow \hat{\mathcal{O}}_{\Sigma} \stackrel{\hat{\bar{\partial}}}{\longrightarrow} \bar{K}_{\hat{\Sigma}} \rightarrow 0
$$

We can define the operator $\hat{\bar{\partial}} f=[\operatorname{dzd} \theta] \bar{D} f, \quad \bar{D} f=\frac{\mathrm{d}}{\mathrm{d} \bar{\theta}}+\bar{\theta} \frac{\mathrm{d}}{\mathrm{d} \bar{z}}, \quad f \in \Gamma\left(\hat{\mathcal{O}}_{\Sigma}\right), \quad(z \mid \theta) \in \hat{\Sigma}$. Note that the operator $\bar{D}$ is $N=1$ supersymmetric anti-holomorphic vector fields. Tensoring this exact sequence with any super line bundles $L_{\hat{\Sigma}}$, we have

$$
0 \rightarrow L_{\hat{\Sigma}} \hookrightarrow L_{\hat{\Sigma}} \otimes \hat{\mathcal{O}}_{\Sigma} \stackrel{\hat{\bar{\partial}}_{L_{\hat{\Sigma}}}}{\longrightarrow} L_{\hat{\Sigma}} \otimes \bar{K}_{\hat{\Sigma}} \rightarrow 0 .
$$

We can define the operator $\hat{\bar{\partial}}_{L_{\hat{\Sigma}}}(f s)=(\hat{\bar{\partial}} f) \cdot s+f \cdot \hat{\bar{\partial}}_{L_{\hat{\Sigma}}} s, \quad f \in \Gamma\left(\hat{\mathcal{O}}_{\Sigma}\right), s \in \Gamma\left(L_{\hat{\Sigma}}\right)$. We can describe $H^{0}\left(\hat{\Sigma}, L_{\hat{\Sigma}}\right)$ as the space of sections $s$ of $L_{\hat{\Sigma}}$ satisfying the condition $\hat{\bar{\partial}}_{L_{\hat{\Sigma}}} s=0$. The group $H^{1}\left(\hat{\Sigma}, L_{\hat{\Sigma}}\right)$ can be described as the space of sections $\Gamma\left(L_{\hat{\Sigma}} \otimes \bar{K}_{\hat{\Sigma}}\right)$ modulo the image of the operator $\hat{\bar{\partial}}_{L_{\hat{\Sigma}}}$. Hence $\operatorname{dimKer} \hat{\bar{\partial}}_{L_{\hat{\Sigma}}}=\operatorname{dim} H^{0}\left(\hat{\Sigma}, L_{\hat{\Sigma}}\right)$ and $\operatorname{dimCoker} \hat{\bar{\partial}}_{L_{\hat{\Sigma}}}=\operatorname{dim} H^{1}\left(\hat{\Sigma}, L_{\hat{\Sigma}}\right)$.

Let $Z_{0}, Z_{1}, \cdots, Z_{r} \in \hat{M}$ be $(r+1)$ distinct points and $Z_{i}=\left(z_{i} \mid \theta_{i}^{1}, \theta_{i}^{2}, \cdots, \theta_{i}^{N}\right), \quad(i=0,1,2, \cdots, r)$. Then the super meromorphic functions

$$
f(Z)=h(Z) \prod_{i=0}^{r}\left(z-Z_{i}-\sum_{\alpha=1}^{N} \theta^{\alpha} \theta_{i}^{\alpha}\right)^{n_{i}}
$$

is coresponding to the super Weil divisor (cf. [18] [20])

$$
D=\sum_{i=0}^{r} n_{i}\left[Z_{i}\right] \text {, }
$$

where $h(Z)$ is a super holomorphic function. We put $x=z-z_{i}-\sum_{\alpha=1}^{N} \theta^{\alpha} \theta_{i}^{\alpha}, \quad x=x_{B}+x_{S}, \quad x_{B}=z-z_{i}$ and $x_{S}=-\sum_{\alpha=1}^{N} \theta^{\alpha} \theta_{i}^{\alpha}$. Then the inverse element of $x$, which is unique, is given by the formula $x^{-1}=x_{B}^{-1} \sum_{n=0}^{N}\left(-x_{B}^{-1} x_{S}\right)^{n}$ (cf. [23]). As an application, we have a main theorem as follows.

Theorem 4.2 Let $\hat{\Sigma}$ be the complex $(1 \mid N)$-dimensional supercurves with genus $g$ and $L_{\hat{\Sigma}}=L_{\Sigma} \otimes \hat{\mathcal{O}}_{\Sigma}$ be any super line bundles of rank (1|0) on $\hat{\Sigma}$. Then we have a Riemann-Roch type formula as follows. 


$$
\operatorname{dim} H^{0}\left(\hat{\Sigma}, L_{\hat{\Sigma}}\right)-\operatorname{dim} H^{1}\left(\hat{\Sigma}, L_{\hat{\Sigma}}\right)=2^{N}\left(\operatorname{ch}\left(L_{\hat{\Sigma}}\right) \cdot \operatorname{td}(T \hat{\Sigma})\right)[\Sigma]
$$

where $[\Sigma] \in H_{2}(\Sigma, \mathbb{Z})$ is the fundamental homology class.

Proof. Let us consider the super divisor $D=1 \cdot\left[Z_{0}\right]$ on $\hat{\Sigma}$. The local equation on $D$ is defined by $s_{i}=z-z_{0}-\sum_{\alpha=1}^{N} \theta^{\alpha} \theta_{0}^{\alpha}$ on a open set $U_{i}$ of $\hat{M}$. If $Z_{0} \in U_{i}$, then $s_{i}\left(Z_{0}\right)=0$. The super Weil divisor can be considered as the super Cartier divisor. Then there is the exact sequence

$$
0 \rightarrow L_{1}^{-1} \stackrel{\varphi}{\longrightarrow} \hat{\mathcal{O}}_{\Sigma} \stackrel{r_{Z_{0}}}{\longrightarrow} \mathcal{L}_{Z_{0}} \rightarrow 0
$$

The line sheaf $L_{1}$ corresponding to $D=1 \cdot\left[Z_{0}\right]$ is defined by the transition functions

$$
g_{i j}=\left(z-z_{0}-\sum_{\alpha=1}^{N} \theta^{\alpha} \theta_{0}^{\alpha}\right)^{-1}
$$

on $U_{i} \cap U_{j}$. The sheaf $L_{1}^{-1}$ which is defined by

$$
\left.L_{1}^{-1}\right|_{U}=\left\{\phi \in O_{\Sigma}(U)|\phi|_{U} \cap\left(1 \cdot\left[Z_{0}\right]\right)=0\right\}
$$

is the coherent ideal sheaf. The fiber $\left.\mathcal{L}_{Z_{0}}\right|_{Z}$ of $\mathcal{L}_{Z_{0}}$ is of zero in $Z \neq Z_{0}$ and $\mathbb{C}^{12^{N}-1}$ in $Z=Z_{0}$. The sheaf $\mathcal{L}_{Z_{0}}$ is called the super skyscraper sheaf. Tensoring this with $L_{1}$, we have

$$
0 \rightarrow \hat{\mathcal{O}}_{\Sigma} \stackrel{\psi}{\longrightarrow} L_{1} \stackrel{r_{Z_{0}}}{\longrightarrow} \mathcal{L}_{Z_{0}} \rightarrow 0 \text {. }
$$

The map $\psi$ is defined by $\left.\psi\right|_{U_{i}}: \mathcal{O}_{U_{i}} \rightarrow \mathcal{O}_{U_{i}}\left(z-z_{0}-\sum_{\alpha=1}^{N} \theta^{\alpha} \theta_{0}^{\alpha}\right)^{-1}$ on an open set $U_{i}$ of $\hat{\Sigma}$. Taking cohomology, this gives a long exact sequence

$$
\begin{aligned}
0 & \rightarrow H^{0}\left(\hat{\Sigma}, \hat{\mathcal{O}}_{\Sigma}\right) \rightarrow H^{0}\left(\hat{\Sigma}, L_{1}\right) \rightarrow H^{0}\left(\hat{\Sigma}, \mathcal{L}_{Z_{0}}\right) \\
& \rightarrow H^{1}\left(\hat{\Sigma}, \hat{\mathcal{O}}_{\Sigma}\right) \rightarrow H^{1}\left(\hat{\Sigma}, L_{1}\right) \rightarrow H^{1}\left(\hat{\Sigma}, \mathcal{L}_{Z_{0}}\right) \rightarrow 0 .
\end{aligned}
$$

Taking the alternative sum, we have

$$
\begin{aligned}
& \operatorname{dim} H^{0}\left(\hat{\Sigma}, \hat{\mathcal{O}}_{\Sigma}\right)-\operatorname{dim} H^{0}\left(\hat{\Sigma}, L_{1}\right)+\operatorname{dim} H^{0}\left(\hat{\Sigma}, \mathcal{L}_{Z_{0}}\right) \\
& -\operatorname{dim} H^{1}\left(\hat{\Sigma}, \hat{\mathcal{O}}_{\Sigma}\right)+\operatorname{dim} H^{1}\left(\hat{\Sigma}, L_{1}\right)-\operatorname{dim} H^{1}\left(\hat{\Sigma}, \mathcal{L}_{Z_{0}}\right)=0 .
\end{aligned}
$$

Noting that $H^{0}\left(\hat{\Sigma}, \mathcal{L}_{Z_{0}}\right)=\mathbb{C}^{12^{N}-1}$ and $H^{1}\left(\hat{\Sigma}, \mathcal{L}_{Z_{0}}\right)=0$, we have

$$
\operatorname{dim} H^{0}\left(\hat{\Sigma}, \hat{\mathcal{O}}_{\Sigma}\right)-\operatorname{dim} H^{1}\left(\hat{\Sigma}, \hat{\mathcal{O}}_{\Sigma}\right)=\operatorname{dim} H^{0}\left(\hat{\Sigma}, L_{1}\right)-\operatorname{dim} H^{1}\left(\hat{\Sigma}, L_{1}\right)-2^{N} .
$$

From $c_{1}\left(L_{1}\right)=1$ and $2^{N}=2^{N} C_{1}\left(L_{1}\right)$, we have

$$
\operatorname{dim} H^{0}\left(\hat{\Sigma}, \hat{\mathcal{O}}_{\Sigma}\right)-\operatorname{dim} H^{1}\left(\hat{\Sigma}, \hat{\mathcal{O}}_{\Sigma}\right)=\operatorname{dim} H^{0}\left(\hat{\Sigma}, L_{1}\right)-\operatorname{dim} H^{1}\left(\hat{\Sigma}, L_{1}\right)-(1+N) c_{1}\left(L_{1}\right) .
$$

We also take the exact sequence

$$
0 \rightarrow L_{\hat{\Sigma}} \rightarrow L_{\hat{\Sigma}} \otimes L_{1} \rightarrow \mathcal{L}_{Z_{0}} \rightarrow 0 .
$$

This gives rise to a long exact sequence

$$
\begin{aligned}
0 & \rightarrow H^{0}\left(\hat{\Sigma}, L_{\hat{\Sigma}}\right) \rightarrow H^{0}\left(\hat{\Sigma}, L_{\hat{\Sigma}} \otimes L_{1}\right) \rightarrow H^{0}\left(\hat{\Sigma}, \mathcal{L}_{Z_{0}}\right) \\
& \rightarrow H^{1}\left(\hat{\Sigma}, L_{\hat{\Sigma}}\right) \rightarrow H^{1}\left(\hat{\Sigma}, L_{\hat{\Sigma}} \otimes L_{1}\right) \rightarrow H^{1}\left(\hat{\Sigma}, \mathcal{L}_{Z_{0}}\right) \rightarrow 0 .
\end{aligned}
$$

Taking also the alternative sum, we have 


$$
\begin{aligned}
& \operatorname{dim} H^{0}\left(\hat{\Sigma}, L_{\hat{\Sigma}}\right)-\operatorname{dim} H^{0}\left(\hat{\Sigma}, L_{\hat{\Sigma}} \otimes L_{1}\right)+\operatorname{dim} H^{0}\left(\hat{\Sigma}, \mathcal{L}_{Z_{0}}\right) \\
& -\operatorname{dim} H^{1}\left(\hat{\Sigma}, L_{\hat{\Sigma}}\right)+\operatorname{dim} H^{1}\left(\hat{\Sigma}, L_{\hat{\Sigma}} \otimes L_{1}\right)-\operatorname{dim} H^{1}\left(\hat{\Sigma}, \mathcal{L}_{Z_{0}}\right)=0 .
\end{aligned}
$$

Hence, we havet

$$
\operatorname{dim} H^{0}\left(\hat{\Sigma}, L_{\hat{\Sigma}}\right)-\operatorname{dim} H^{1}\left(\hat{\Sigma}, L_{\hat{\Sigma}}\right)=\operatorname{dim} H^{0}\left(\hat{\Sigma}, L_{\hat{\Sigma}} \otimes L_{1}\right)-\operatorname{dim} H^{1}\left(\hat{\Sigma}, L_{\hat{\Sigma}} \otimes L_{1}\right)-2^{N} .
$$

Note that $c_{1}\left(L_{\hat{\Sigma}}\right)+c_{1}\left(L_{1}\right)=c_{1}\left(L_{\hat{\Sigma}} \otimes L_{1}\right)$. So adding $-2^{N} c_{1}\left(L_{\hat{\Sigma}}\right)$ in both side, we see that

$$
\begin{aligned}
& \operatorname{dim} H^{0}\left(\hat{\Sigma}, L_{\hat{\Sigma}}\right)-\operatorname{dim} H^{1}\left(\hat{\Sigma}, L_{\hat{\Sigma}}\right)-2^{N} c_{1}\left(L_{\hat{\Sigma}}\right) \\
& =\operatorname{dim} H^{0}\left(\hat{\Sigma}, L_{\hat{\Sigma}} \otimes L_{1}\right)-\operatorname{dim} H^{1}\left(\hat{\Sigma}, L_{\hat{\Sigma}} \otimes L_{1}\right)-2^{N} c_{1}\left(L_{\hat{\Sigma}} \otimes L_{1}\right) .
\end{aligned}
$$

Therefore, $\operatorname{dim} H^{0}\left(\hat{\Sigma}, L_{\hat{\Sigma}}\right)-\operatorname{dim} H^{1}\left(\hat{\Sigma}, L_{\hat{\Sigma}}\right)-2^{N} c_{1}\left(L_{\hat{\Sigma}}\right)$ is independent of $L_{\hat{\Sigma}}$, so that we can put $L_{\hat{\Sigma}}=\hat{\mathcal{O}}_{\Sigma}$. From Theorem 6.1, $\operatorname{dim} H^{0}\left(\hat{\Sigma}, \hat{\mathcal{O}}_{\Sigma}\right)-\operatorname{dim} H^{1}\left(\hat{\Sigma}, \hat{\mathcal{O}}_{\Sigma}\right)-2^{N} c_{1}\left(\hat{\mathcal{O}}_{\Sigma}\right)=2^{N} t d_{1}(T \hat{\Sigma})$. This completes the proof of Theorem 4.2.

\section{Helicity Group}

Definition 5.1 The helicity rank of finitely generated group $G$ is defined by the positive generator of linearly independent itself. The helicity rank is denoted by $\operatorname{rank}_{\mathrm{h}} G$. The helicity rank of $\Pi G$ is defined by the negative generator of linearly independent itself. The helicity rank of $G \oplus G$ also is defined by twice the positive generator of linearly independent itself of $G$.

We define the finitely generated group of two type as follows.

$$
\begin{aligned}
& \frac{1}{n} \mathbb{Z}=\left\{\frac{1}{n} a \mid a \in \mathbb{Z}, n \in \mathbb{Z}: \text { fix }\right\}, \\
& \Pi \mathbb{Z}=\{\Pi 0=0, \Pi( \pm 1)=\mp 1, \Pi( \pm 2)=\mp 2, \Pi( \pm 3)=\mp 3, \cdots\} .
\end{aligned}
$$

Note that $\Pi \mathbb{Z}, \frac{1}{n} \mathbb{Z}$ and $\Pi\left(\frac{1}{n} \mathbb{Z}\right)$ are isomorphic to $\mathbb{Z}, \mathbb{Z}$ and $\frac{1}{n} \mathbb{Z}$ as abelian groups, respectively. But its helicity rank is differently as follows.

Example 5.1 $\operatorname{rank}_{\mathrm{h}} \mathbb{Z}=1$, $\operatorname{rank}_{\mathrm{h}} \mathbb{Z} \oplus \mathbb{Z}=2, \operatorname{rank}_{\mathrm{h}} \mathbb{Z}_{m}=0$,

$$
\begin{aligned}
& \operatorname{rank}_{\mathrm{h}}\left(\frac{1}{n} \mathbb{Z}\right)=\frac{1}{n}, \operatorname{rank}_{\mathrm{h}}(n \mathbb{Z})=n, \operatorname{rank}_{\mathrm{h}}(\Pi \mathbb{Z})=-1, \operatorname{rank}_{\mathrm{h}}(\Pi \mathbb{Z} \oplus \Pi \mathbb{Z})=-2, \\
& \operatorname{rank}_{\mathrm{h}}\left(\Pi\left(\frac{1}{n} \mathbb{Z}\right)\right)=-\frac{1}{n}, \operatorname{rank}_{\mathrm{h}}\left(\frac{1}{n} \mathbb{Z}\right)^{\oplus N}=\frac{N}{n}, \operatorname{rank}_{\mathrm{h}}\left(\Pi\left(\frac{1}{n} \mathbb{Z}\right)^{\oplus N}\right)=-\frac{N}{n} .
\end{aligned}
$$

Definition 5.2 Let $\hat{M}=\left(M, \hat{\mathcal{O}}_{M}\right)$ be a $(n \mid N)$-dimensional complex supermanifold. Then the helicity group $\mathbb{G}_{(n \mid N)}$ is defined by the following.

$$
\mathbb{G}_{(n \mid N)}=Z \oplus \Pi\left(\frac{1}{n+1} Z\right)^{\oplus N}, \quad \mathbb{G}_{(n \mid 0)}=Z, \quad \mathbb{G}_{(0 \mid N)}=\Pi Z^{\oplus N} .
$$

The helicity rank of $\mathbb{G}_{(n \mid N)}$ can be represented by

$$
\operatorname{rank}_{\mathrm{h}} \mathbb{G}_{(n \mid N)}=1-\frac{N}{n+1} .
$$

The super cohomology with coefficient in $\mathbb{G}_{(n \mid N)}$ of an $(n \mid N)$-dimensional supermanifold $\left(\hat{M}, \hat{\mathcal{O}}_{M}\right)$ is 
isomorphic to the $\mathbb{G}_{(n \mid N)}$-valued cohomology with coefficient in $\mathbb{Z}$ of the classical manifold $M$ using the universal coefficient theorem. That is to say, we have the following.

$$
H^{i}\left(\hat{M}, \mathbb{G}_{(n \mid N)}\right) \simeq H^{i}(M, \mathbb{Z}) \otimes \mathbb{G}_{(n \mid N)} \simeq\left(H^{i}(M, \mathbb{Z}) \otimes \mathbb{Z}\right) \oplus\left(H^{i}(M, \mathbb{Z}) \otimes \Pi\left(\frac{1}{n+1} \mathbb{Z}\right)^{\oplus N}\right) .
$$

This isomrphism is applied in section 6 .

\section{Gauss-Bonnet Type Formula}

In this section, we will apply the super cohomology with coefficient in helicity group $\mathbb{G}_{(n \mid N)}$.

Theorem 6.1 Let $\hat{\Sigma}$ be the complex $(1 \mid N)$-dimensional supercurves with genus $g$. Then we have a GaussBonnet type formula as follows.

$$
e(T \hat{\Sigma})=\sum_{i=0}^{2}(-1)^{i} \operatorname{rank}_{\mathrm{h}} H^{i}\left(\hat{\Sigma}, \mathbb{Z} \oplus \Pi\left[\left(\frac{1}{2} \mathbb{Z}\right)^{\oplus N}\right]\right) .
$$

Proof. Euler number of $T \hat{\Sigma}$ get

$$
e(T \hat{\Sigma})=c_{1}(T \hat{\Sigma})=c_{1}(T \Sigma)-c_{1}\left(\left[K_{\Sigma}^{-\frac{1}{2}}\right]^{\oplus N}\right)=(2-2 g)-N(1-g)=(2-N)(1-g) .
$$

Note that $c_{1}\left(K_{\hat{\Sigma}}\right)=-c_{1}(T \hat{\Sigma})$. On the other hand, the right hand side is

$$
\left(1-\frac{N}{2}\right)-2 g\left(1-\frac{N}{2}\right)+\left(1-\frac{N}{2}\right)=(2-N)(1-g) \text {. }
$$

Both sides coincide.

Theorem 6.2 Let $P^{n \mid N}$ be the complex $(n \mid N)$-dimensional super projective space. Then, we have

$$
c_{1}\left(T P^{n \mid N}\right)=\sum_{i=0}^{2 n}(-1)^{i} \operatorname{rank}_{\mathrm{h}} H^{i}\left(P^{n \mid N}, \mathbb{Z} \oplus \Pi\left[\left(\frac{1}{n+1} \mathbb{Z}\right)^{\oplus N}\right]\right) .
$$

\section{Proof.}

From the super Euler sequence, we can compute the total Chern class of holomorphic tangent bundle $T P^{n \mid N}$. Setting $\mathcal{O}(d)=\mathcal{O}_{P^{n \mid N}}(d)$ for simplicity's sake and $x=c_{1}(\mathcal{O}(1)) \in H^{2}\left(P^{n}, \mathbb{Z}\right) \otimes \mathbb{G}_{(n \mid N)}$, we have

$$
\begin{aligned}
& c\left(T P^{n \mid N}\right)=c\left(\mathbb{C}^{n+1 \mid N} \otimes \mathcal{O}(1)\right)=c(\underbrace{\mathcal{O}(1) \oplus \cdots \oplus \mathcal{O}(1)}_{(n+1) \text {-times }} \oplus \underbrace{\Pi \mathcal{O}(1) \oplus \cdots \oplus \Pi \mathcal{O}(1)}_{N \text {-times }}) \\
& =c(\mathcal{O}(1) \oplus \cdots \oplus \mathcal{O}(1)) \mid c(\Pi \mathcal{O}(1) \oplus \cdots \oplus \mathcal{O}(1))=(1+x)^{n+1}(1-x)^{N} \\
& =\left(1+(n+1) x+\left(\begin{array}{c}
n+1 \\
2
\end{array}\right) x^{2}+\cdots+(n+1) x^{n}\right) \times\left(1-N x+\left(\begin{array}{c}
N \\
2
\end{array}\right) x^{2}-\left(\begin{array}{c}
N \\
3
\end{array}\right) x^{3}+\cdots\right)
\end{aligned}
$$

The sum of coefficient of $x$ is the first super Chern number $c_{1}\left(T P^{n \mid N}\right)$.

$$
\begin{aligned}
c_{1}\left(T P^{n \mid N}\right) & =(n+1)-N=(n+1)\left(1-\frac{N}{n+1}\right) \\
& =\sum_{i=0}^{2 n} \operatorname{rank}_{h}\left(H^{i}\left(P^{n}, \mathbb{Z}\right) \otimes \mathbb{G}_{(n \mid N)}\right) \\
& =\sum_{i=0}^{2 n} \operatorname{rank}_{h} H^{i}\left(P^{n \mid N}, \mathbb{G}_{(n \mid N)}\right)=\text { right hand side }
\end{aligned}
$$




\section{References}

[1] Taniguchi, T. (2009) ADHM Construction of Super Yang-Mills Instantons. Journal of Geometry and Physics, 59, 11991209. http://dx.doi.org/10.1016/j.geomphys.2009.06.003

[2] Bartocci, C., Bruzzo, U. and Ruipérez, D.H. (1991) The Geometry of Supermanifolds. Mathematics and Its Applications, Volume 71, Kluwer Academic Publishers, Norwell.

[3] LeBrun, C., Poon, Y.S. and Wells Jr., R.O. (1990) Projective Embedding of Complex Supermanifolds. Communications in Mathematical Physics, 126, 433-452. http://dx.doi.org/10.1007/BF02125694

[4] Leites, D.A. (1980) Introduction to the Theory of Supermanifolds. Russian Mathematical Surveys, 35, 1-64.

[5] Manin, Yu.I. (1997) Gauge Field Theory and Complex Geometry. 2nd Edition, Springer, Berlin. http://dx.doi.org/10.1007/978-3-662-07386-5

[6] Rogers, A. (2007) Supermanifolds Theory and Applications. World Scientific, Singapore City. http://dx.doi.org/10.1142/9789812708854

[7] LeBrun, C. and Rothstein, M. (1988) Moduli of Super Riemann Surfaces. Communications in Mathematical Physics, 117, 159-176. http://dx.doi.org/10.1007/BF01228415

[8] Penkov, I.B. (1983) D-Modules on Supermanifolds. Inventiones Mathematicae, 71, 501-512. http://dx.doi.org/10.1007/BF02095989

[9] Bartocci, C. and Bruzzo, U. (1988) Cohomology of the Structure Sheaf of Real and Complex Supermanifolds. Journal of Mathematical Physics, 29, 1789-1794. http://dx.doi.org/10.1007/BF02095989

[10] Bott, R. and Tu, L.W. (1982) Differential Forms in Algebraic Topology. Springer, Berlin. http://dx.doi.org/10.1007/978-1-4757-3951-0

[11] Bruzzo, U. and Ruipérez, D.H. (1989) Characteristic Classes of Super Vector Bundles. Journal of Mathematical Physics, 30, 1233-1237. http://dx.doi.org/10.1063/1.528606

[12] Hartshorne, R. (1977) Algebraic Geometry. Springer, Berlin. http://dx.doi.org/10.1007/978-1-4757-3849-0

[13] Hirzebruch, F. (1966) Topological Methods in Algebraic Geometry. Springer-Verlag, Berlin.

[14] Lawson Jr., H.B. and Michelsohn, M. (1989) Spin Geometry. Princeton University Press, Princeton.

[15] Voronov, A.A. and Manin, Y.I. (1990) Elements of Supergeometry. Journal of Mathematical Sciences, 51, $2069-2083$.

[16] Bruzzo, U. and Fucito, F. (2004) Superlocalization Formulas and Supersymmetric Yang-Mills Theories. Nuclear Physics B, 678, 638-655. http://dx.doi.org/10.1016/j.nuclphysb.2003.11.033

[17] Ninnemann, H. (1992) Deformations of Super Riemann Surfaces. Communications in Mathematical Physics, 150, 267288. http://dx.doi.org/10.1007/BF02096661

[18] Rosly, A.A., Schwarz, A.S. and Voronov, A.A. (1988) Geometry of Superconformal Manifolds. Communications in Mathematical Physics, 119, 129-152. http://dx.doi.org/10.1007/BF01218264

[19] Crane, L. and Rabin, J.M. (1988) Super Riemann Surfaces: Uniformization and Teichmüller Theory. Communications in Mathematical Physics, 113, 601-623. http://dx.doi.org/10.1007/BF01223239

[20] Manin, Y.I. (1991) Topics in Non-Commutative Geometry. M. B. Porter Lectures at Rice University, Houston. http://dx.doi.org/10.1515/9781400862511

[21] Giddings, S.B. and Nelson, P. (1988) The Geometry of Super Riemann Surfaces. Communications in Mathematical Physics, 116, 607-634. http://dx.doi.org/10.1007/BF01224903

[22] Giddings, S.B. and Nelson, P. (1988) Line Bundles on Super Riemann Surfaces. Communications in Mathematical Physics, 118, 289-302. http://dx.doi.org/10.1007/BF01218581

[23] De Witt, B. (1992) Supermanifolds. 2nd Edition, Cambridge University Press, Cambridge. http://dx.doi.org/10.1017/CBO9780511564000 